\title{
A kinase-independent biological activity for insulin growth factor-1 receptor (IGF-1R): implications for inhibition of the IGF-1R signal
}

\author{
Filip Janku' ${ }^{1}$, Helen J. Huang ${ }^{1}$, Laura S. Angelo ${ }^{1}$, and Razelle Kurzrock ${ }^{2}$ \\ ${ }^{1}$ Department of Investigational Cancer Therapeutics (Phase I Clinical Trials Program), The University of Texas MD Anderson \\ Cancer Center, Houston, Texas \\ ${ }^{2}$ Moores Cancer Center, The University of California San Diego, La Jolla, California \\ Correspondence to: Filip Janku, email: fjanku@mdanderson.org \\ Keywords: IGF-1R, OSI-906, intracellular glucose, autophagy, MCF7 cells \\ Received: February 17, $2013 \quad$ Accepted: March 23, 2013 \\ Published: March 23, 2013
}

This is an open-access article distributed under the terms of the Creative Commons Attribution License, which permits unrestricted use, distribution, and reproduction in any medium, provided the original author and source are credited.

\section{ABSTRACT:}

It has been demonstrated that epidermal growth factor receptor (EGFR) can have kinase independent activity. EGFR kinase-independent function maintains intracellular glucose levels via sodium glucose transporter protein 1 (SGLT1) and supports cell survival. It is plausible that this phenomenon can apply to other receptor tyrosine kinases. We found that transfection of insulin-like growth factor receptor (IGF-1R) siRNA into HEK293 (human embryonic kidney) and MCF7 (metastatic breast cancer) cells results in decreased intracellular glucose levels, whereas treatment with the IGF-1R tyrosine kinase inhibitor OSI-906 did not affect intracellular glucose levels. In addition, IGF-1R interacted with SGLT1 in a manner similar to that previously reported with EGFR. The combination of IGF-1R siRNA and OSI-906 resulted in decreased viability of HEK293 and MCF7 cell lines compared to either agent alone. Collectively, these experiments suggest that IGF-1R, has kinase-independent biologic functions and provide a rationale for combining anti-IGF-1R antibodies or siRNA and IGF-1R small molecule inhibitors.

\section{INTRODUCTION}

Insulin-like growth factor-1 receptor (IGF1R) signaling is an important metabolic pathway in cancer.[1] IGF-1R inhibitors have shown promise in the clinic in several different malignancies, including Ewing's sarcoma, non-small cell lung carcinoma, and adrenocortical carcinoma.[2-5] Both anti-IGF-1R antibodies and small molecules directed against the kinase activity of IGF-1R are being assessed in clinical trials.[4, 6-13] Anti-IGF-1R antibodies down-regulate cell-surface IGF-1R, thereby preventing signaling through IGF-1R. Small molecule kinase inhibitors, such as OSI-906, inhibit the phosphorylation and downstream signaling of IGF-1R. [14] While these molecules target two distinct points in the IGF-1 signaling pathway, the end result is thought to be the same, i.e. down-regulation of signaling through IGF-1R.

A recent publication demonstrated that epidermal growth factor receptor (EGFR) and other kinases signal through both kinase-dependent and kinase-independent pathways.[15, 16]. For instance, down-regulation of EGFR expression with small interfering (si) RNA resulted in a decrease in intracellular glucose levels, and induction of autophagy, as well as an increase in the sub-G1 population in metastatic prostate cancer cells.[16-18]. These observations have important clinical implications as they suggest that mitigating EGFR signaling may require a combination of small molecule (kinase) inhibitors and antibodies (or siRNA). Indeed, synergy between an EGFR kinase inhibitor and an antibody has been demonstrated in preclinical models, and is being tested in the clinic.[19-23] It is plausible that this phenomenon may not be unique to EGFR and can apply to other transmembrane kinase receptors. In our study, we explored whether IGF-1R also demonstrated kinase-independent activity similar to that found with EGFR. 


\section{RESULTS}

\section{IGF-1R siRNA decreases total IGF-1R, p-IGF-1R, and $p$ - $A k t$}

HEK293 and MCF7 cells, which both express high levels of IGF-1R, were transfected with siRNA against IGF-1R, and Western blotting was performed to confirm the effect of down-regulation of IGF-1R siRNA on p-IGF$1 \mathrm{R}$ and p-Akt.[24, 25]. IGF-1R siRNA decreased the expression of total IGF-1R and p-IGF-1R in both cells lines (Figure 1a.), and phosphorylation of the downstream effector Akt was inhibited in HEK293 cells. In MCF7 cells, Akt phosphorylation was not downregulated despite a decrease in total and p-IGF-1R levels, most likely because Akt activation is induced in these cells via both IGF-1R and the insulin receptor (IR).[24]. Transfection with EGFR siRNA served as a control for the specificity of IGF-1R siRNA. GAPDH siRNA served as a positive control for transfection (Figure 1a).

\section{OSI-906 decreases p-IGF-1R and p-Akt}

OSI-906 is a dual kinase inhibitor that inhibits both IGF-1R and IR signaling. MCF7 cells express both IGF$1 R$ and IR (and also IR-A, the fetal isoform of IR).[24] Hence, IGF-1R siRNA was not sufficient to decrease p-Akt in MCF7 cells, but OSI-906 was (Figure 1b). This is consistent with data in the literature suggesting that Akt activation in MCF7 cells involves both IGF-1R and
IR. Total IGF-1R remains constant following OSI-906 treatment in both cell lines, while p-Akt and p-IGF-1R are decreased in a dose-dependent manner (Figure 1b).

\section{IGF-1R siRNA decreases intracellular glucose levels}

Previously, transfection of EGFR siRNA into metastatic prostate cancer cells resulted in decreased intracellular glucose levels, whereas treatment with an EGFR kinase inhibitor did not affect intracellular glucose. [16]. GLUT is a facilitative glucose transporter, and SGLT1 is an active glucose transporter (sodium/glucose cotransporter [SGLT]). These proteins allow glucose to move into and out of a cell.[26, 27] HEK293 and MCF7 cells were transfected with non-targeted, IGF1R, SGLT1, or GLUT siRNA, and intracellular glucose levels were measured (Figures $2 a$ and $2 b$ ). In both cell lines, intracellular glucose decreased significantly after transfection with IGF-1R siRNA. This was demonstrated in HEK293 at low/physiological extracellular glucose levels $(5 \mathrm{mM})$, and was reversible at high $(25 \mathrm{mM})$ glucose levels (Figure 2a). In MCF7 cells, transfection of IGF-1R siRNA decreased intracellular glucose levels at both physiological and high glucose levels (Figure 2b), but was more pronounced at low glucose levels. SGLT1 and GLUT siRNA also decreased intracellular glucose concentrations in both cell lines, and like IGF-1R siRNA, the effect was only seen at low extracellular glucose concentrations in HEK293, and was less pronounced in MCF7 cells.

\section{a. SIRNA}

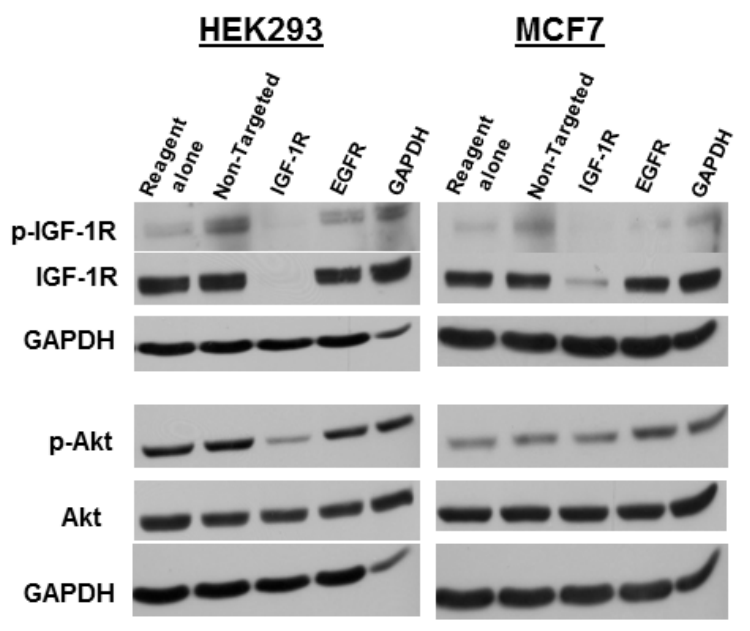

b. OSI-906

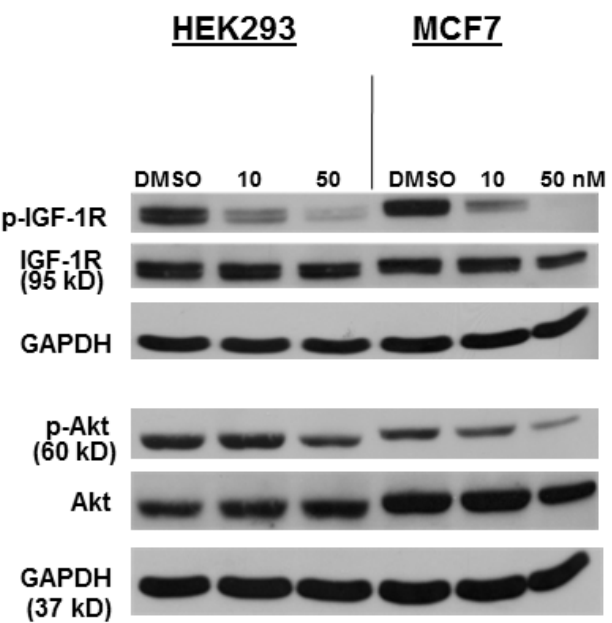

Figure 1: The effect of IGF-1R siRNA or OSI-906 on p-IGF-1R and p-Akt. (a) IGF-1R siRNA decreases total IGF-1R and p-IGF-1R in HEK293 and MCF7 cells, and p-Akt in HEK293 cells. Cells were transfected with $100 \mathrm{nM}$ siRNA for 48 hours, then lysed for Western blot analysis using appropriate antibodies. EGFR siRNA was used as a control for the specificity of IGF-1R siRNA, and GAPDH siRNA was used as a positive control for transfection. (b) The small molecule kinase inhibitor, OSI-906, inhibits phosphorylation of IGF$1 \mathrm{R}$ and Akt in HEK293 and MCF7 cells in a dose-dependent manner. Total IGF-1R levels remain unchanged. Cells were treated for 48 hours, then lysed for Western blotting. GAPDH was used as a loading control. Blots are representative of at least three experiments. 
In contrast to IGF-1R siRNA, OSI-906 did not decrease intracellular glucose levels under any conditions (low 5mM, medium $17 \mathrm{mM}$, or high $25 \mathrm{mM}$ extracellular glucose) when tested at concentrations up to $1 \mathrm{uM}$ (Figure 2c). Concentrations of $50 \mathrm{nM}$ OSI-906 demonstrated complete (MCF7) or near complete (HEK293) inhibition of IGF-1R kinase activity (Figure 1b), but intracellular glucose levels remained unchanged. These results are analogous to those obtained with EGFR siRNA versus EGFR kinase inhibitors, and indicate that inhibition of the kinase domain of IGF-1R has no effect on the levels of intracellular glucose in either cell line, while downregulation of total IGF-1R decreases intracellular glucose. [16]

\section{IGF-1R and SGLT1 interact in HEK293 and MCF7 cells}

In the paper by Weihua et al., EGFR interacted with SGLT1, but not with GLUT, in PC3MM2 cells.[16] We performed immuno-precipitation followed by Western blot analysis to determine if IGF-1R and SGLT1 also interact endogenously in our cell lines (Figure 3a). When HEK293 or MCF7 cell lysates were immuno-precipitated with antiSGLT1 and Western blotted with anti-IGF-1R, a strong band appeared (Figure 3a, top, lane 2). This was also true when the antibodies were reversed (Figure $3 \mathrm{a}$, bottom, lane 2). GLUT, however, does not appear to interact with IGF-1R or EGFR (Figure 3a, top and middle panels, lane 3). Cell lysates were immuno-precipitated with antiEGFR and Western blotted with anti-SGLT1 as a positive control for interaction with SGLT1 (Figure 3a, bottom, lane 3).[16]. In addition to immuno-precipitation/Western blot, interaction of IGF-1R and SGLT-1 was implicated by experiments in which transfection of both cell lines with IGF-1R siRNA also resulted in a decrease in SGLT1 protein expression, whereas non-targeted siRNA did not have this effect (Figure 3b, compare lanes 3 and 4). This is similar to results obtained for EGFR in that downregulation of total EGFR by siRNA transfection resulted in decreased expression of SGLT1 at the protein level. a.

$\underline{\text { HEK293 }}$

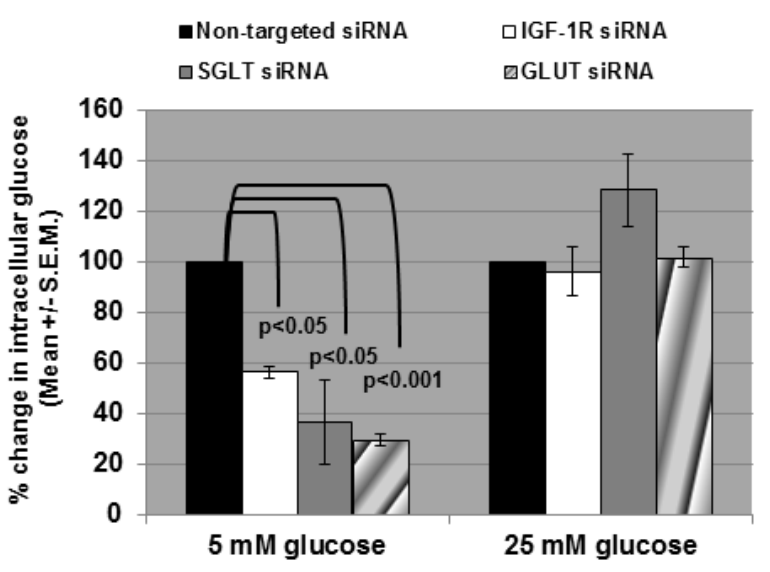

b.

\section{$\underline{\text { MCF7 }}$}
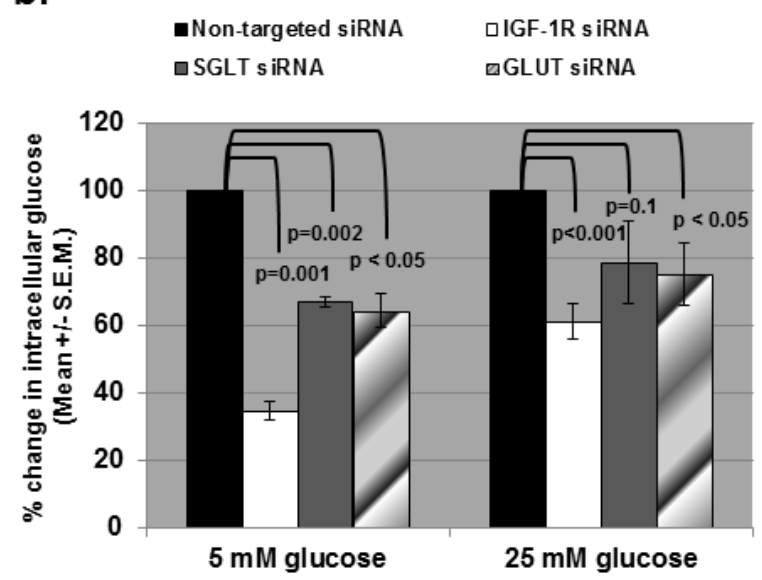

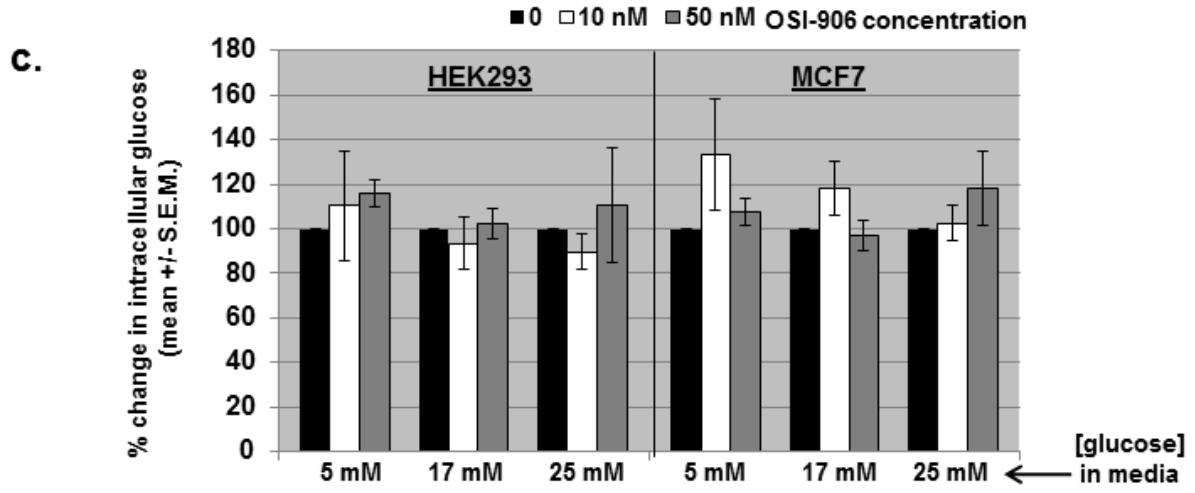

Figure 2: siRNA against IGF-1R, SGLT, and GLUT decrease intracellular glucose levels, but OSI-906 does not. (a) IGF-1R, SGLT, and GLUT siRNA significantly decreased intracellular glucose levels in HEK293 cells cultured in low/physiologic (5mM) glucose DMEM, but not in high $(25 \mathrm{mM})$ glucose DMEM. (b) IGF-1R, SGLT, and GLUT siRNA significantly decreased intracellular glucose levels in MCF7 cells cultured in low glucose, and to a lesser extent in high glucose. (c) Intracellular glucose levels were not significantly affected by OSI-906 (10-50 nM) in either cell line in low, medium $(17 \mathrm{mM})$, or high $(25 \mathrm{mM})$ glucose DMEM. All experiments were repeated at least three times and significance determined by student's test. 
[16]

\section{IGF-1R down-regulation does not result in autophagy in HEK293 and MCF7 cells}

Since siRNA against EGFR was found to induce autophagic cell death in PC3MM2 cells, we asked whether this was unique to EGFR or if down-regulation of IGF-1R by siRNA could also induce autophagy.[16]. Autophagy typically occurs when the cell is under environmental stress, such as nutrient deprivation, or pathogen infection, and is used to degrade and recycle proteins and organelles as a means of survival, and may result in increased or decreased cell death depending on the circumstances. $[17,18]$. Autophagy induction was a possibility since levels of intracellular glucose were decreased by IGF-1R siRNA transfection in a manner similar to that observed for EGFR.[16]. However, IGF-1R siRNA did not induce autophagy in either HEK293 or MCF7 cells. Cells were transfected with non-targeted or IGF-1R siRNA and then immuno-stained for the presence of isoform II of the autophagic protein, microtubule-associated protein 1 light chain 3 (LC3II) (Figure 4a and 4b). Cells were analyzed by flow cytometry. LC3B isoform II typically increases in cells that are undergoing autophagy. Neither IGF-1R siRNA nor OSI-906 induced autophagy in either cell line. MG132, a proteasome inhibitor, was used as a positive inducer of autophagy to show that both cell lines can be induced to increase LC3II expression (Figure 4a and 4b, right panels) even though MCF7 lacks beclin1, a protein important to the autophagic process.[28, 29]. Results were confirmed by Western blot (Figure $4 \mathrm{c}$ and $4 \mathrm{~d}$ ). Therefore, unlike EGFR, IGF-1R down-regulation did not result in autophagy in these two cell lines. Interestingly, OSI-906 actually decreased the level of LC3II by Western blotting in both cell lines. This result was seen consistently in at least four experiments, indicating that OSI-906 may in fact inhibit autophagy in these cells. Cell cycle analysis was also performed to determine if IGF-1R siRNA increased the sub-G1 population (dead/apoptotic cells) as was found for EGFR. Results showed that IGF-1R siRNA did not increase the sub-G1 population in either cell line (Supplementary Figure 1).

\section{IGF-1R siRNA decreases the viability of HEK293 and MCF7 cells alone and in combination with OSI-906}

We next determined whether IGF-1R siRNA and OSI-906 affected the viability of the cells either alone or in combination. Cells treated with $0.1 \%$ DMSO were assigned $100 \%$ viability and were used as a negative (vehicle) control. Cells treated with media alone were virtually identical to DMSO only treated cultures. Prior to assessing the effect on viability, the IC50 of OSI-906 on both cell lines was determined (approximately $3 \mathrm{uM}$ a. HEK293

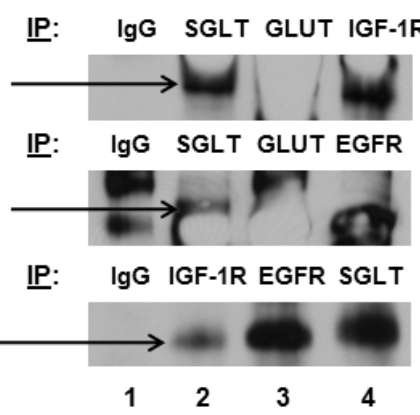

b.

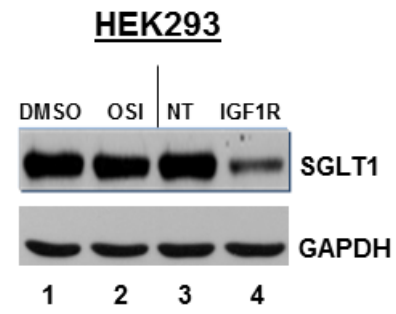

MCF7

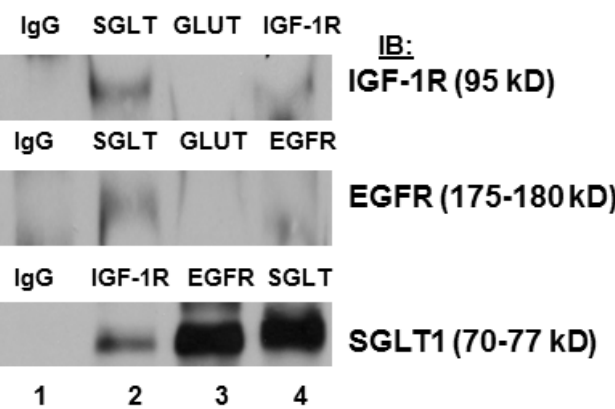

$\underline{\text { MCF7 }}$

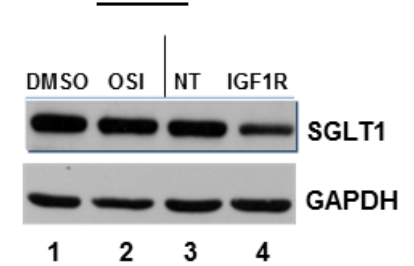

Figure 3: IGF-1R and SGLT1 interact in HEK293 and MCF7 cells, and IGF-1R siRNA transfection results in downregulation of SGLT1. (a) HEK293 and MCF7 cells were lysed with RIPA buffer, and 500-1000 ug of protein was immuno-precipitated with IgG isotypic control, anti-SGLT1, anti-GLUT, or anti-IGF-1R plus protein A/G beads. Immuno-precipitated extracts were run on an $8 \%$ SDS-PAGE gel, immuno-blotted, and probed with anti-IGF-1R, anti-EGFR, or anti-SGLT1. Experiment shown is representative of at least three experiments. Arrows indicate the interaction. (b) HEK293 and MCF7 cells were treated with vehicle (DMSO), 1 uM OSI-906, or transfected with non-targeted siRNA or IGF-1R siRNA for 48 hours. Inhibition of IGF-1R with siRNA also decreases protein expression of SGLT1 (lane 4), whereas OSI-906 has no effect on the expression of SGLT1. GAPDH served as a loading control. 
for HEK293 at 96 hours and 500-750 nM for MCF7 at 96 hours and $1 \mathrm{uM}$ for MCF7 at 72 hours). Hence all viability and apoptosis experiments were carried out at 1 uM OSI-906 at 72 or 96 hours. A cell viability assay was used to measure the number of viable cells present based on quantification of ATP, which signals the presence of metabolically active cells. The amount of ATP is directly proportional to the number of cells present in culture (see Materials and Methods). Cells were either treated with DMSO as a control (100\% viability) or DMSO plus non-targeted siRNA, DMSO plus IGF-1R siRNA, OSI-906 alone, OSI-906 plus non-targeted siRNA, or OSI-906 plus IGF-1R siRNA. Treatment with 1 uM OSI-906 significantly decreased the viability of HEK 293 and MCF7 cells by approximately $26 \%(\mathrm{p}<0.05)$ and $50 \%(\mathrm{p}<0.001)$, respectively, at 72 hours and by $30 \%$ $(\mathrm{p}<0.05)$ and $52 \%(\mathrm{p}<0.001)$, respectively, at 96 hours (Figure 5, compare bars A and B). Hence, OSI-906 was more effective at decreasing the viability of MCF7 cells than the viability of HEK293 cells at $1 \mathrm{uM}$. These data correlate with the previously calculated IC50 for each cell line. IGF-1R siRNA alone decreased the viability of HEK 293 and MCF7 cells by $28 \%(\mathrm{p}<0.05)$ and $10 \%$ $(\mathrm{p}<0.05)$, respectively, at 72 hours and $40 \%(\mathrm{p}<0.05)$ and $15 \%(\mathrm{p}<0.05)$ at 96 hours (Figure 5, compare bars A and E). Hence, down-regulation of total IGF-1R by siRNA transfection resulted in a statistically significant decrease in the viability of HEK293 cells and MCF7 cells at both time points. Cells were also transfected with non-targeted siRNA as a negative control. There was a non-specific decrease in the viability of both cell lines following transfection of non-targeted siRNA, but this was not statistically significant (Figure 5, compare bars A and C).

The inhibitory effect of OSI-906 alone compared with IGF-1R siRNA alone varied with the cell line and the amount of time of culture. In HEK293 cells, OSI-906 and
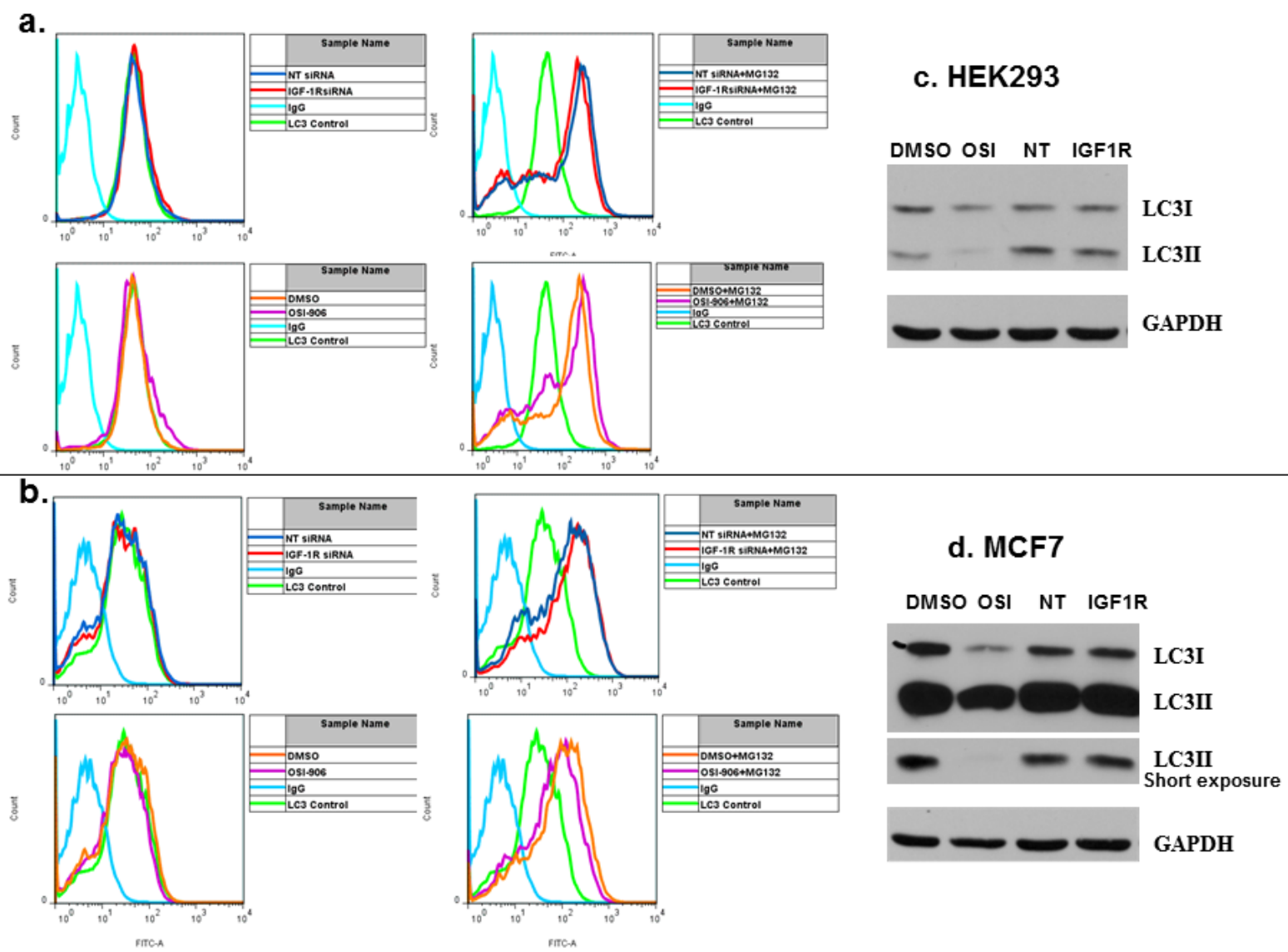

Figure 4: Neither IGF-1R siRNA nor OSI-906 induces the autophagic protein LC3II in HEK293 or MCF7 cells. HEK293 and MCF7 cells were transfected with non-targeted or IGF-1R siRNA for 48 hours (top panels, a. and b.) or treated with 1 uM OSI-906 for 48 hours (lower panels, a. and b.). Cells were washed in PBS and immuno-stained with primary anti-LC3B antibody, then stained with FITC-conjugated secondary antibody and subjected to FACS analysis. An IgG antibody was used as an isotypic control (light blue); baseline anti-LC3B staining (light green); non-targeted siRNA (dark blue); IGF-1R siRNA (red); DMSO (orange); OSI-906 (light

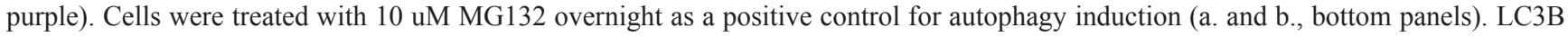
isoforms I and II were also analyzed by Western blotting following transfection of HEK293 (c) and MCF7 (d) with siRNA or treatment with $1 \mathrm{uM}$ OSI-906 for $48 \mathrm{hrs}$. GAPDH expression served as a loading control. NT = non-targeted siRNA. 
IGF-1R siRNA did not differ in their ability to decrease viability at 72 hours, but IGF-1R siRNA was more effective than OSI-906 if cultured for 96 hours (Figure $5 \mathrm{a}$ and 5b, compare bars B and E). In MCF7 cells, OSI906 was much more effective $(p<0.001$, Figure $5 c$ and $5 d$, compare A and B) than IGF-1R siRNA $(p<0.05$, Figure $5 \mathrm{c}$ and $5 \mathrm{~d}$, compare $\mathrm{A}$ and $\mathrm{E}$ ) at decreasing viability at 72 and 96 hours (compare bars B and E), with an enhanced decrease at 96 hours (Figure 5c and 5d)

If IGF-1R has both kinase-dependent and kinaseindependent effects, and if these effects are pertinent to cell viability, then the combination of IGF-1R siRNA plus OSI-906 would be expected to decrease the viability of both cell lines more than either agent alone. Figure 5 shows the effect on the viability of HEK293 and MCF7 cells treated with the combination of OSI-906 plus IGF-1R siRNA. Comparison of OSI-906 alone to treatment with OSI-906 plus IGF-1R siRNA (Figure 5, bar B compared to bar F) shows a significant enhancement of the effects of OSI-906 in both cell lines after transfection with IGF-1R siRNA at 72 hours and even more so at 96 hours (HEK293 $\mathrm{p}<0.05$ at 72 hours, $\mathrm{p}<0.001$ at 96 hours; MCF7 $\mathrm{p}<0.05$ at 72 hours, $\mathrm{p}<0.001$ at 96 hours). Alternatively, comparison of DMSO plus IGF-1R siRNA (essentially IGF-1R siRNA alone) to OSI-906 plus IGF-1R siRNA (Figure 5, compare bar $\mathrm{E}$ to bar F) also shows a significant decrease in viability of both cell lines at 72 hours (HEK293 p $<0.05$, MCF7 p<0.001) and 96 hours (HEK293 p<0.05, MCF7 $\mathrm{p}<0.001)$. Comparing OSI-906 plus non-targeted siRNA to OSI-906 plus IGF-1R siRNA (Figure 5 compare bar D and F) shows that IGF-1R siRNA is significantly better than non-targeted siRNA when combined with OSI-906 in HEK293 cells at 72 hours $(\mathrm{p}<0.05)$ and MCF7 at 96 hours $(\mathrm{p}<0.001)$, and shows a trend toward significance in MCF7 cells at 72 hours $(\mathrm{p}=0.07)$. A significant difference between the two siRNAs was not seen in HEK293 cells at 96 hours,
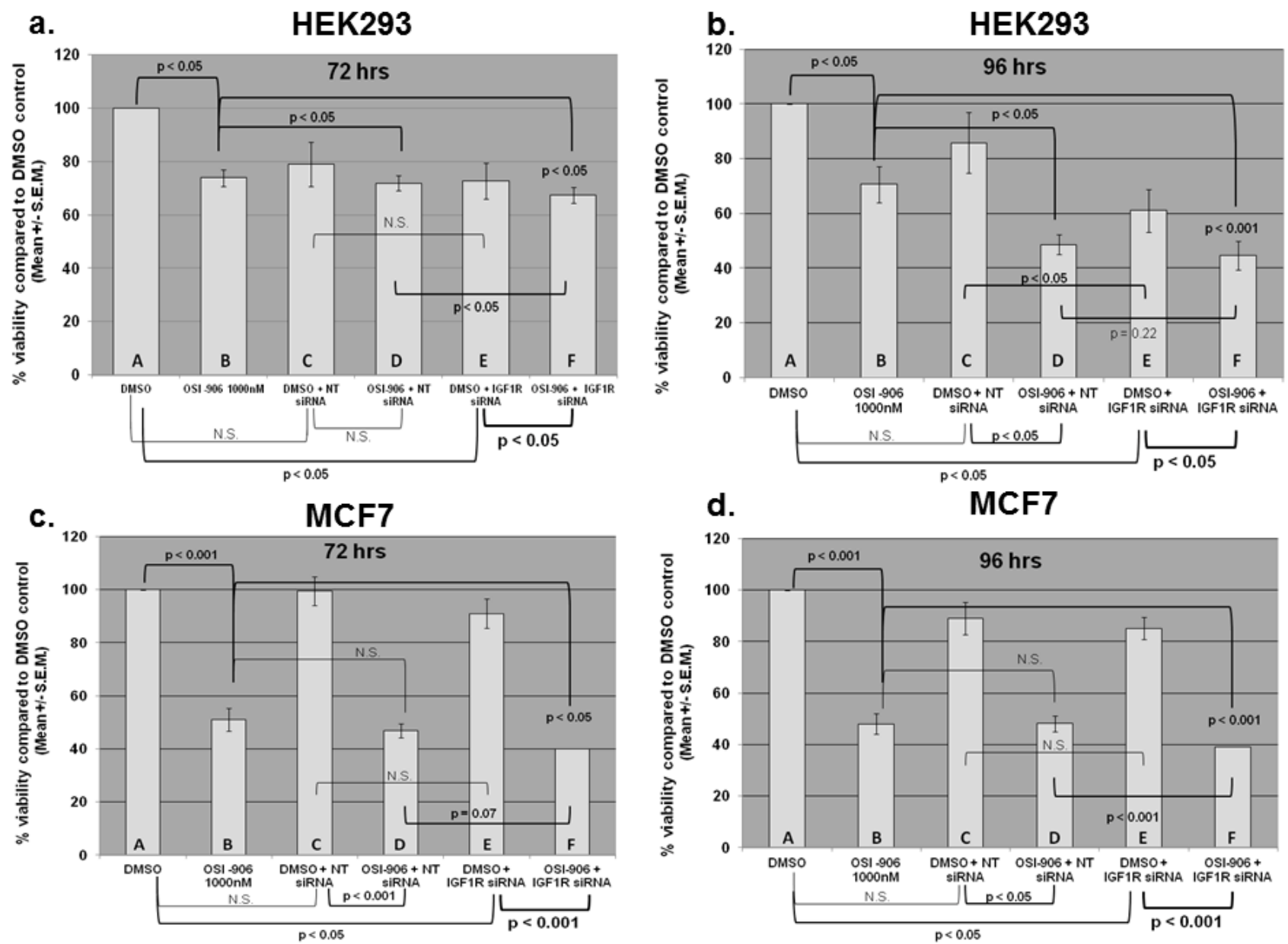

d.

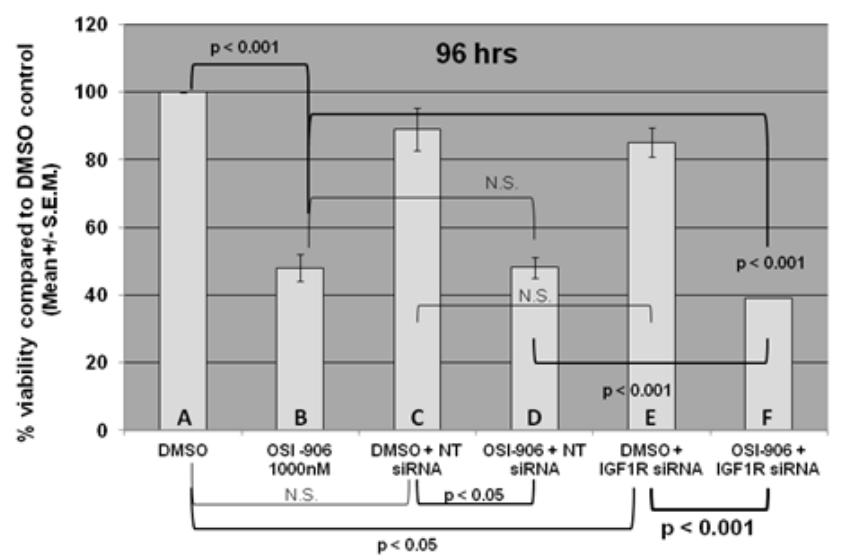

Figure 5: Viability assay results for HEK293 (low glucose) and MCF7 (high glucose) cells transfected with non-targeted or IGF-1R siRNA alone, treated with OSI-906 alone, or various combinations as indicated. Cells were transfected with either non-targeted or IGF-1R siRNA $(100 \mathrm{nM})$ for 24 hours, then treated with OSI-906 (1 uM) for an additional 48 or 72 hours, giving final incubation times of 72 or 96 hours. (a) HEK293 - 72 hours; (b) HEK293 - 96 hours; (c) MCF7 - 72 hours; (d) MCF7 - 96 hours. Capital letters were added to the bar graphs for ease of comparison. $\mathrm{A}=\mathrm{DMSO}, \mathrm{B}=1 \mathrm{uM}$ OSI-906 alone, $\mathrm{C}=\mathrm{DMSO}+$ non-targeted siRNA, $\mathrm{D}=$ OSI-906 + NT siRNA, E= DMSO + IGF-1R siRNA, F= OSI-906 + IGF-1R siRNA. Statistical differences were determined by Student's t test. $\mathrm{P}$ values $<0.05$ were considered significant. 
perhaps because the S.E.M. was larger for this time point. Therefore, these results suggest that the combination of the kinase inhibitor OSI-906 and siRNA against IGF-1R has significantly greater suppressive effects on HEK293 and MCF7 viability than either agent alone, though these effects are more pronounced at certain time points.

\section{OSI-906 and IGF-1R siRNA do not induce apoptosis or increase the sub-G1 population in HEK293 or MCF7 cells}

EGFR siRNA increased the $\mathrm{subG}_{1}$ population in transfected metastatic prostate cancer cells [14]. Hence, we treated our cell lines with OSI-906 alone, IGF-1R siRNA alone, or the combination and performed cell cycle analysis. As stated above, there was no significant increase in the subG1 population in either cell line after treatment with OSI-906 or IGF-1R siRNA (Supplementary Figure 1). FITC-Annexin V staining was also performed to determine if our cell lines were undergoing apoptosis after treatment with OSI-906 alone or in combination with IGF-1R siRNA. Baseline levels of apoptosis were slightly higher in MCF7 cells (22\%). Treatment of MCF7 with OSI-906 induced only a slight increase in apoptosis (4\%) over baseline, and the combination of OSI-906 plus IGF-1R siRNA resulted in a 7\% increase in apoptosis compared to OSI-906 alone (not significant by Student's t test, Supplementary Figure 2). Baseline levels of apoptosis were lower in HEK293 cells (10\%) and increased to approximately $18 \%$ after treatment with OSI-906 alone and increased to only $20 \%$ with the combination (not statistically significant by Student's t test, Supplementary Figure 3). Neither OSI-906, IGF-1R siRNA, nor the combination significantly induced apoptosis in MCF7 and HEK293 cells. Together, these data indicate that the combination of OSI-906 and IGF-1R siRNA inhibits proliferative pathways, but does not induce apoptotic cell death in the cell lines studied.

\section{DISCUSSION}

Receptor kinases can signal through more than one pathway. For example, CRAF was found to have an MEK-independent role, which is critical for mitosis and tumor progression.[15]. In addition, Weihua et al. [16] demonstrated that siRNA directed against EGFR resulted not only in the down-regulation of total EGFR, but also in a decrease in intracellular glucose levels, the induction of autophagic cell death, and an increase in the sub-G1 population. These results were not observed when EGFR kinase activity was inhibited, consistent with the presence of a kinase-independent EGFR signal. Additional preclinical data examining EGFR includes animal models showing that the combination of antiEGFR antibodies plus EGFR tyrosine kinase inhibitors, such as erlotinib, results in synergistic tumor regression. [19-21]. We therefore asked whether a kinase-independent signal could be observed with other clinically relevant growth factor receptors, such as IGF-1R. Indeed, recent literature suggests that IGF-1R translocates to the nucleus where it is thought to be involved in the transcriptional enhancement of specific target genes, while the kinase domain of IGF-1R is responsible for interaction with other kinases and the activation of intracellular signaling pathways that result in increased proliferation.[30-36]

Our data demonstrate that siRNA directed against total IGF-1R decreases intracellular glucose levels in both HEK293 (human embryonic kidney) and MCF7 (metastatic breast cancer) cell lines, particularly at physiological levels of glucose $(5 \mathrm{mM})$. Higher levels of extracellular glucose can partially attenuate this effect (Figure 2a and b). OSI-906, a small molecule inhibitor of the IGF-1R kinase, did not alter intracellular glucose levels. In addition, IGF-1R was found to interact with the sodium glucose transporter protein SGLT1 in a manner similar to that seen with EGFR. Hence, IGF-1R (like EGFR) may play a role in maintaining intracellular glucose levels in tumor cells in a kinase-independent manner.

In contrast to EGFR, blocking total IGF-1R expression by siRNA transfection did not increase the sub-G1 (dead) cell population, nor did it induce autophagy or apoptosis in either cell line following inhibition of IGF$1 \mathrm{R}$ expression; however, decreased intracellular glucose levels are not always a prerequisite for autophagy.[37] Even so, the combination of OSI-906 plus IGF-1R siRNA resulted in a greater decrease in cell viability in both cell lines than with either molecule alone (Figure 5). This may have clinical implications in that it suggests that giving an IGF-1R antibody or siRNA together with OSI-906 may be more effective than either agent alone. Of interest, Zeng et al. demonstrated an increase in autophagy in breast cancer cell line models (MCF-7, LCC6) sequentially exposed to doxorubicin and IGF-1R/IR tyrosine kinase inhibitor; however, autophagy in these experiments could have been activated by doxorubicin rather than IGF-1R inhibition. [38]

The case for using a monoclonal antibody and a kinase inhibitor together is being studied in the clinic. $[22,23]$. We recently demonstrated that 11 of 34 patients (32.4\%) achieved stable disease for at least six months and/or a partial/complete response with the use of the EGFR kinase inhibitor erlotinib combined with the EGFR antibody cetuximab.[39]. Others have shown that combining the HER2/neu kinase inhibitor lapatinib with a HER2/neu antibody trastuzumab prolonged progressionfree survival in patients with HER2/neu positive breast cancer.[40, 41]. Our current results suggest that a similar strategy may be exploitable with IGF-1R inhibition.

There are limitations to this study. For instance, OSI-906 inhibits insulin receptor kinase as well as IGF-1R 
kinase. It is therefore possible that the more pronounced decrease in cell viability observed when it was combined with siRNA against IGF-1R could be due to the effects on the insulin receptor itself. Still, these results suggest that such a combination in the clinic may be worth examining.

In summary, we show that IGF-1R, like EGFR, demonstrates a kinase-independent function. The kinaseindependent activity is related to the interaction of IGF-1R with the sodium glucose transporter SGLT1; indeed, down-regulation of IGF-1R suppresses SGLT1, thereby attenuating glucose levels. Unlike EGFR, down-regulation of IGF-1R by siRNA does not result in increased autophagy. However, cell viability is decreased and, furthermore, combining the kinase inhibitor OSI-906 with siRNA against IGF-1R amplifies the suppression of viability, albeit without a significant pro-apoptotic effect. These observations suggest that combining an IGF-1R siRNA (or antibody) with OSI-906 is warranted in the clinical setting. Finally, experiments are underway to determine if other tyrosine kinase receptors also have kinase-independent functions.

\section{MATERIALS AND METHODS}

\section{Cell lines}

HEK293 (human embryonic kidney) and MCF7 (breast adenocarcinoma) cell lines were obtained from American Type Culture Collection (ATCC, Manassas, VA), and were authenticated by short tandem repeat analyses. Both cell lines were maintained in Eagle's Minimal Essential medium (EMEM) (ATCC) plus 10\% fetal calf serum (FCS) and maintained in a $37^{\circ} \mathrm{C}$ incubator with $5 \% \mathrm{CO}_{2}$. For cultures grown in low $(5 \mathrm{mM})$ or high (25 mM) glucose, HyClone low or high glucose DMEM were used, respectively (LabSource, Romeoville, IL).

\section{Antibodies and reagents}

Primary antibodies used for Western blotting included rabbit polyclonal or murine monoclonal antibody against IGF-1R, phosphorylated (p)-IGF-1R (Tyr 1135/1136), Light Chain 3B (LC3B), EGFR, Akt, and p-Akt (ser 473) (Cell Signaling, Danvers, MA), glucose transporter (GLUT1), SGLT1 (Abcam, Cambridge, MA and Millipore, Billerica, MA), and GAPDH (Santa Cruz Biotechnology, Santa Cruz, CA). Secondary antibodies for Western blotting included anti-rabbit (Cell Signaling) and anti-mouse (BioRad Laboratories, Hercules, CA) conjugated to horse radish peroxidise (HRP). OSI-906 was purchased from ChemieTek (Indianapolis, IN). Glucose stock (225 mM) was prepared (Invitrogen, Carlsbad, CA) and diluted with culture media.

\section{siRNA transfection}

siRNA transfection was performed to determine the effect of down-regulating IGF-1R and other molecules on cell signaling, survival, autophagy, apoptosis, and intracellular glucose levels. HEK293 $\left(2.5 \times 10^{5}\right)$ or MCF7 $\left(5 \times 10^{5}\right)$ cells were seeded per well of a 6 well plate and transfected with DharmaFECT 1 siRNA lipid transfection reagent alone (Dharmacon, Pittsburgh, PA), non-targeted siRNA (siGENOME non-targeting siRNA \#1), IGF-1R, EGFR, SGLT, GLUT, or GAPDH siRNA (control) for 48 hours. Following transfection, cell lysates were harvested for Western blot analysis, prepared for an intracellular glucose assay, analyzed by fluorescence activated cell sorter (FACS) analysis, or harvested for viability assay.

\section{Intracellular glucose assay}

Intracellular glucose levels were measured following transfection of cells with IGF-1R siRNA or treatment with OSI-906 to determine the effects of IGF-1R down-regulation or inhibition of IGF-1R tyrosine kinase activity on intracellular glucose levels. $1 \times 10^{4}$ cells per $\mathrm{mL}$ were lysed in Western blotting solubilization buffer and mixed with assay reagent (1.5 mM NAD, $1.0 \mathrm{mM}$ ATP, $1.0 \mathrm{unit} / \mathrm{mL}$ of hexokinase, and $1.0 \mathrm{unit} / \mathrm{mL}$ of glucose- 6 phosphate dehydrogenase) according to the manufacturer's instructions (Glucose [HK] assay kit, Sigma, St. Louis, MO). When glucose is phosphorylated by ATP, an equimolar amount of NAD is reduced to NADH. The consequent increase in absorbance at $340 \mathrm{~nm}$ of the lysate is directly proportional to glucose concentration.

\section{Immuno-precipitation and Western blotting}

Immuno-precipitation followed by Western blotting was performed to determine if there was a physical interaction between IGF-1R and glucose transporter proteins. For Western blots, cells were washed with phosphate buffered saline (PBS) and lysed in 1x RIPA Lysis Buffer (Millipore) plus protease inhibitors. A modified Lowry protein assay was performed (Pierce Biotechnology, Rockford, IL), and equal amounts of protein were loaded onto 7.5-10\% SDS-PAGE gels, and then transferred to nitrocellulose membranes by electroblotting. Blots were blocked in 5.0\% non-fat milk, incubated with primary antibody $\left(22^{\circ} \mathrm{C}\right.$ for 2 hours), washed, and then incubated with an appropriate secondary antibody conjugated to HRP and detected by enhanced chemiluminescence (Amersham Pharmacia Biotech, Piscataway, NJ). For immuno-precipitation experiments, cells were lysed in RIPA buffer (Pierce Biotechnology). 500-1000 ug per lane of lysate was pre-cleared with an isotypic control (Santa Cruz Biotechnology) $\left(4^{\circ} \mathrm{C}\right.$ for 2 
hours), then incubated with specific antibody plus protein A/G beads (2-4 hours at $\left.4^{\circ} \mathrm{C}\right)$, centrifuged, washed $4 \mathrm{X}$ with RIPA buffer, resuspended in 2X sample buffer (BioRad), and boiled before loading onto SDS-PAGE gels.

\section{Analysis of autophagy by LC3B staining and flow cytometry}

To determine if cells were undergoing autophagy, levels of the autophagosomal protein LC3B isoform II were measured by staining the cells with an anti-LC3B antibody, and then analyzing by flow cytometry. HEK293 $\left(2.5 \times 10^{5}\right)$ and MCF7 $\left(5 \times 10^{5}\right)$ cells were transfected with the appropriate siRNA or treated with DMSO (vehicle) or OSI-906 for 48 hours, washed in PBS, and fixed by adding $2-4 \%$ formaldehyde solution for 10 minutes at $37^{\circ} \mathrm{C}$. Cells were then permeabilized by adding ice-cold 100\% methanol to pre-chilled cells to a final concentration of $90 \%$ and incubated on ice for 30 minutes. $0.5-1 \times 10^{6}$ cells per tube were washed twice in $0.5 \%$ bovine serum albumin (BSA) in PBS. Cells were blocked in $0.5 \%$ BSA for 10 minutes at room temperature (RT). Primary antibody (antiLC3B) was added at a 1:500 dilution and incubated for 1 hour at RT, then washed with $0.5 \%$ BSA. FITC-conjugated secondary antibody $(1: 1,000)$ was added and cells were incubated for 30 minutes at RT, washed, and resuspended in $0.5 \mathrm{~mL}$ PBS and analyzed by fluorescence activated cell sorter (FACS) analysis (Becton Dickinson, Bedford, MA).

\section{Viability assay}

Viability of the cells was assessed after treating the cells with OSI-906, IGF-1R siRNA, or the combination. 500 HEK293 or $1 \times 10^{3}$ MCF7 cells were plated per well of an opaque-walled 96-well plate. The CellTiter-Glo® Luminescent Cell Viability Assay (Promega, Madison, WI) was used to measure the number of viable cells present based on quantification of ATP, which signals the presence of metabolically active cells. The amount of ATP is directly proportional to the number of cells present in culture. The assay was performed at RT as described in the manufacturer's instructions. Background luminescence from wells containing media alone was subtracted from experimental wells. $100 \mathrm{uL}$ of CellTiter-Glo ${ }^{\circledR}$ Reagent was added per well containing $100 \mathrm{uL}$ of culture medium. Contents were mixed on an orbital shaker for 2 minutes to induce cell lysis, and the plate was incubated at RT for 10 minutes to stabilize the luminescent signal. Plates were read on a BioTek Synergy 4 plate reader (Winooski, VT).

\section{Annexin V apoptosis assay}

Following transfection with IGF-1R siRNA or treatment with OSI-906 or the combination, apoptosis was measured by staining cell-surface phosphatidyl serine with FITC-conjugated Annexin V. 2.5x10 HEK293 and $5 \times 10^{5}$ MCF7 cells were seeded per well of a six-well plate and treated for 72 hours, harvested, and stained with FITC-Annexin V and propidium iodide according to the manufacturer's instructions (BD Pharmingen). Cells were analyzed using fluorescence activated cell sorter (FACS) analysis (Becton Dickinson).

\section{Cell cycle analysis}

Cell cycle analysis was performed to determine if there was an increase in the sub-G1(dead cell) population following transfection with IGF-1R siRNA or treatment with OSI-906. Cells were fixed in 100\% ethanol and stained with propidium iodide, according to the manufacturer's instructions (BD Pharmingen), and then analyzed using FACS analysis.

\section{Statistical analysis}

Student's t-test was used to assess the association between continuous variables. All tests were two-sided, and $P$ values less than 0.05 were considered statistically significant. If not otherwise indicated, error bars in all experiments represent standard deviation error (SE). All statistical analyses were carried out using SPSS 19 computer software (SPSS Chicago, IL).

\section{Conflict of interest}

Filip Janku has research support from Novartis. Razelle Kurzrock has research support from Glaxo Smith Kline, Novartis, Merck, and Bayer.

\section{REFERENCES}

1. Shaw LM. The insulin receptor substrate (IRS) proteins: at the intersection of metabolism and cancer. Cell Cycle. 2011; 10(11):1750-1756.

2. Tolcher AW, Sarantopoulos J, Patnaik A, Papadopoulos K, Lin CC, Rodon J, Murphy B, Roth B, McCaffery I, Gorski KS, Kaiser B, Zhu M, Deng H, Friberg G and Puzanov I. Phase I, pharmacokinetic, and pharmacodynamic study of AMG 479, a fully human monoclonal antibody to insulin-like growth factor receptor 1. J Clin Oncol. 2009; 27(34):5800-5807.

3. Rodon J, DeSantos V, Ferry RJ, Jr. and Kurzrock R. Early drug development of inhibitors of the insulin-like growth factor-I receptor pathway: lessons from the first clinical trials. Mol Cancer Ther. 2008; 7(9):2575-2588.

4. Kurzrock R, Patnaik A, Aisner J, Warren T, Leong S, Benjamin R, Eckhardt SG, Eid JE, Greig G, Habben K, McCarthy CD and Gore L. A phase I study of weekly 
R1507, a human monoclonal antibody insulin-like growth factor-I receptor antagonist, in patients with advanced solid tumors. Clin Cancer Res. 2010; 16(8):2458-2465.

5. Yee D. Insulin-like growth factor receptor inhibitors: baby or the bathwater? J Natl Cancer Inst. 2012; 104(13):975981.

6. Jassem J, Langer CJ, Karp DD, Mok T, Benner RJ, Green SJ, Park K, Novello S, Strausz J and Gualberto A. Randomized, open label, phase III trial of figitumumab in combination with paclitaxel and carboplatin versus paclitaxel and carboplatin in patients with non-small cell lung cancer (NSCLC). J Clin Oncol. 2010; 28(15s):abstr 7500 .

7. Molife LR, Fong PC, Paccagnella L, Reid AH, Shaw HM, Vidal L, Arkenau HT, Karavasilis V, Yap TA, Olmos D, Spicer J, Postel-Vinay S, Yin D, Lipton A, Demers L, Leitzel K, et al. The insulin-like growth factor-I receptor inhibitor figitumumab (CP-751,871) in combination with docetaxel in patients with advanced solid tumours: results of a phase Ib dose-escalation, open-label study. Br J Cancer. 2010; 103(3):332-339.

8. Bagatell R, Herzog CE, Trippett TM, Grippo JF, Cirrincione-Dall G, Fox E, Macy M, Bish J, Whitcomb P, Aikin A, Wright G, Yurasov S, Balis FM and Gore L. Pharmacokinetically guided phase 1 trial of the IGF-1 receptor antagonist RG1507 in children with recurrent or refractory solid tumors. Clin Cancer Res. 2011; 17(3):611619.

9. Reidy DL, Vakiani E, Fakih MG, Saif MW, Hecht JR, Goodman-Davis N, Hollywood E, Shia J, Schwartz J, Chandrawansa K, Dontabhaktuni A, Youssoufian H, Solit DB and Saltz LB. Randomized, phase II study of the insulin-like growth factor-1 receptor inhibitor IMC-A12, with or without cetuximab, in patients with cetuximab- or panitumumab-refractory metastatic colorectal cancer. J Clin Oncol. 2010; 28(27):4240-4246.

10. Naing A, Kurzrock R, Burger A, Gupta S, Lei X, Busaidy N, Hong D, Chen HX, Doyle LA, Heilbrun LK, Rohren E, Ng C, Chandhasin C and LoRusso P. Phase I trial of cixutumumab combined with temsirolimus in patients with advanced cancer. Clin Cancer Res. 2011; 17(18):60526060

11. Ekman S, Frodin JE, Harmenberg J, Bergman A, Hedlund A, Dahg P, Alvfors C, Stahl B, Bergstrom S and Bergqvist M. Clinical Phase I study with an Insulin-like Growth Factor-1 receptor inhibitor: experiences in patients with squamous non-small cell lung carcinoma. Acta Oncol. 2011; 50(3):441-447.

12. Olmos D, Postel-Vinay S, Molife LR, Okuno SH, Schuetze SM, Paccagnella ML, Batzel GN, Yin D, Pritchard-Jones K, Judson I, Worden FP, Gualberto A, Scurr M, de Bono JS and Haluska P. Safety, pharmacokinetics, and preliminary activity of the anti-IGF-1R antibody figitumumab (CP751,871) in patients with sarcoma and Ewing's sarcoma: a phase 1 expansion cohort study. Lancet Oncol. 2010;
11(2):129-135.

13. Haluska P, Worden F, Olmos D, Yin D, Schteingart D, Batzel GN, Paccagnella ML, de Bono JS, Gualberto A and Hammer GD. Safety, tolerability, and pharmacokinetics of the anti-IGF-1R monoclonal antibody figitumumab in patients with refractory adrenocortical carcinoma. Cancer Chemother Pharmacol. 2010; 65(4):765-773.

14. Mulvihill MJ, Cooke A, Rosenfeld-Franklin M, Buck E, Foreman K, Landfair D, O'Connor M, Pirritt C, Sun Y, Yao Y, Arnold LD, Gibson NW and Ji QS. Discovery of OSI-906: a selective and orally efficacious dual inhibitor of the IGF-1 receptor and insulin receptor. Future Med Chem. 2009; 1(6):1153-1171.

15. Mielgo A, Seguin L, Huang M, Camargo MF, Anand S, Franovic A, Weis SM, Advani SJ, Murphy EA and Cheresh DA. A MEK-independent role for CRAF in mitosis and tumor progression. Nat Med. 2011; 17(12):1641-1645.

16. Weihua Z, Tsan R, Huang WC, Wu Q, Chiu CH, Fidler IJ and Hung MC. Survival of cancer cells is maintained by EGFR independent of its kinase activity. Cancer Cell. 2008; 13(5):385-393.

17. Janku F, McConkey DJ, Hong DS and Kurzrock R. Autophagy as a target for anticancer therapy. Nat Rev Clin Oncol. 2011; 8(9):528-539.

18. Mathew R, Karantza-Wadsworth V and White E. Role of autophagy in cancer. Nat Rev Cancer. 2007; 7(12):961-967.

19. Matar P, Rojo F, Cassia R, Moreno-Bueno G, Di Cosimo S, Tabernero J, Guzman M, Rodriguez S, Arribas J, Palacios $\mathrm{J}$ and Baselga J. Combined epidermal growth factor receptor targeting with the tyrosine kinase inhibitor gefitinib (ZD1839) and the monoclonal antibody cetuximab (IMC-C225): superiority over single-agent receptor targeting. Clin Cancer Res. 2004; 10(19):6487-6501.

20. Huang S, Armstrong EA, Benavente S, Chinnaiyan P and Harari PM. Dual-agent molecular targeting of the epidermal growth factor receptor (EGFR): combining anti-EGFR antibody with tyrosine kinase inhibitor. Cancer Res. 2004; 64(15):5355-5362.

21. Brand TM, Dunn EF, Iida M, Myers RA, Kostopoulos KT, Li C, Peet CR and Wheeler DL. Erlotinib is a viable treatment for tumors with acquired resistance to cetuximab. Cancer Biol Ther. 2011; 12(5):436-446.

22. Ramalingam S, Forster J, Naret C, Evans T, Sulecki M, Lu H, Teegarden P, Weber MR and Belani CP. Dual inhibition of the epidermal growth factor receptor with cetuximab, an IgG1 monoclonal antibody, and gefitinib, a tyrosine kinase inhibitor, in patients with refractory non-small cell lung cancer (NSCLC): a phase I study. J Thorac Oncol. 2008; 3(3):258-264.

23. Janjigian YY, Azzoli CG, Krug LM, Pereira LK, Rizvi NA, Pietanza MC, Kris MG, Ginsberg MS, Pao W, Miller VA and Riely GJ. Phase I/II trial of cetuximab and erlotinib in patients with lung adenocarcinoma and acquired resistance to erlotinib. Clin Cancer Res. 2011; 17(8):2521-2527. 
24. Ulanet DB, Ludwig DL, Kahn CR and Hanahan D. Insulin receptor functionally enhances multistage tumor progression and conveys intrinsic resistance to IGF-1R targeted therapy. Proc Natl Acad Sci U S A. 2010; 107(24):10791-10798.

25. Huang HJ, Angelo LS, Rodon J, Sun M, Kuenkele KP, Parsons HA, Trent JC and Kurzrock R. R1507, an antiinsulin-like growth factor-1 receptor (IGF-1R) antibody, and EWS/FLI-1 siRNA in Ewing's sarcoma: convergence at the IGF/IGFR/Akt axis. PLoS One. 2011; 6(10):e26060.

26. Wood IS and Trayhurn P. Glucose transporters (GLUT and SGLT): expanded families of sugar transport proteins. Br J Nutr. 2003; 89(1):3-9.

27. Ganapathy V, Thangaraju M and Prasad PD. Nutrient transporters in cancer: relevance to Warburg hypothesis and beyond. Pharmacol Ther. 2009; 121(1):29-40.

28. Janen SB, Chaachouay $\mathrm{H}$ and Richter-Landsberg C. Autophagy is activated by proteasomal inhibition and involved in aggresome clearance in cultured astrocytes. Glia. 2010; 58(14):1766-1774.

29. Liang XH, Jackson S, Seaman M, Brown K, Kempkes B, Hibshoosh $\mathrm{H}$ and Levine B. Induction of autophagy and inhibition of tumorigenesis by beclin 1. Nature. 1999; 402(6762):672-676.

30. McElroy B, Powell JC and McCarthy JV. The insulin-like growth factor 1 (IGF-1) receptor is a substrate for gammasecretase-mediated intramembrane proteolysis. Biochem Biophys Res Commun. 2007; 358(4):1136-1141.

31. Sehat B, Tofigh A, Lin Y, Trocme E, Liljedahl U, Lagergren $\mathrm{J}$ and Larsson O. SUMOylation mediates the nuclear translocation and signaling of the IGF-1 receptor. Sci Signal. 2010; 3(108):ra10.

32. Aleksic T, Chitnis MM, Perestenko OV, Gao S, Thomas PH, Turner GD, Protheroe AS, Howarth M and Macaulay VM. Type 1 insulin-like growth factor receptor translocates to the nucleus of human tumor cells. Cancer Res. 2010; 70(16):6412-6419.

33. Levine AJ, Harris CR and Puzio-Kuter AM. The Interfaces Between Signal Transduction Pathways: IGF-1/mTor, p53 and the Parkinson Disease Pathway. Oncotarget. 2012; 3(11):1301-1307.

34. Ucar DA, Kurenova E, Garrett TJ, Cance WG, Nyberg C, Cox A, Massoll N, Ostrov DA, Lawrence N, Sebti SM, Zajac-Kaye M and Hochwald SN. Disruption of the protein interaction between FAK and IGF-1R inhibits melanoma tumor growth. Cell Cycle. 2012; 11(17):3250-3259.

35. Hart LS, Dolloff NG, Dicker DT, Koumenis C, Christensen JG, Grimberg A and El-Deiry WS. Human colon cancer stem cells are enriched by insulin-like growth factor-1 and are sensitive to figitumumab. Cell Cycle. 2011; 10(14):2331-2338.

36. Liu B, Fan Z, Edgerton SM, Yang X, Lind SE and Thor AD. Potent anti-proliferative effects of metformin on trastuzumab-resistant breast cancer cells via inhibition of erbB2/IGF-1 receptor interactions. Cell Cycle. 2011;
10(17):2959-2966.

37. Ravikumar B, Stewart A, Kita H, Kato K, Duden R and Rubinsztein DC. Raised intracellular glucose concentrations reduce aggregation and cell death caused by mutant huntingtin exon 1 by decreasing mTOR phosphorylation and inducing autophagy. Hum Mol Genet. 2003; 12(9):985994.

38. Zeng X, Zhang H, Oh A, Zhang Y and Yee D. Enhancement of doxorubicin cytotoxicity of human cancer cells by tyrosine kinase inhibition of insulin receptor and type I IGF receptor. Breast Cancer Res Treat. 2012; 133(1):117-126.

39. Falchook GS, Wheler JJ, Naing A, Hong DS, Moulder SL, Piha-Paul SA, Ng CS, Jackson E and Kurzrock R. A phase I study of bevacizumab in combination with sunitinib, sorafenib, and erlotinib plus cetuximab, and trastuzumab plus lapatinib. J Clin Oncol. 2010; 28(15s):abstr 2512.

40. Blackwell KL, Burstein HJ, Storniolo AM, Rugo H, Sledge G, Koehler M, Ellis C, Casey M, Vukelja S, Bischoff J, Baselga J and O'Shaughnessy J. Randomized study of Lapatinib alone or in combination with trastuzumab in women with ErbB2-positive, trastuzumab-refractory metastatic breast cancer. J Clin Oncol. 2010; 28(7):11241130.

41. Wu Y, Amonkar MM, Sherrill BH, O’Shaughnessy J, Ellis C, Baselga J, Blackwell KL and Burstein HJ. Impact of lapatinib plus trastuzumab versus single-agent lapatinib on quality of life of patients with trastuzumab-refractory HER2+ metastatic breast cancer. Ann Oncol. 2011; 22(12):2582-2590. 